\title{
Time-dependent prediction of the unsteady pressure near-field from an under-expanded jet
}

\author{
Aldo Rona, Danilo Di Stefano, Alessandro Mancini ${ }^{\dagger}$ and Edward Hall ${ }^{\ddagger}$ \\ Department of Engineering, University of Leicester, Leicester, LE1 7RH, England
}

\begin{abstract}
The near field of an under-expanded air jet issuing from an axi-symmetric convergent nozzle is investigated by Implicit Large Eddy Simulation at a nozzle pressure ratio of 2.27. Estimates are obtained of the tonal components of the unsteady pressure at locations representative of the engine to fuselage distance in a civil transport aircraft. At this relatively short distance from the jet axis, the radiating acoustic pressure field combines with the 'hydrodynamic' pressure perturbations from the jet shear-layer structures, resulting in a combined aerodynamic and acoustic forcing pressure field. The predictions offer useful insight for predicting cabin noise at cruise in civil transport aircraft fitted with high-bypass ratio engines.
\end{abstract}

\section{Introduction}

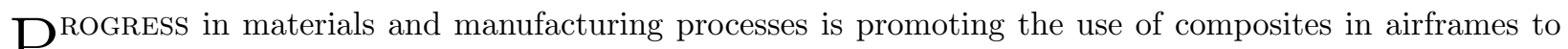
Pachieve reductions in aircraft weight, fuel consumption, and therefore in operating costs. Composites in airframes have been used as secondary structure elements for over 40 years, such as the cabin floor of the Boeing 747 Classic. More recently, composite main structures in transport aircraft have been produced, such as the composite wing box of the Airbus A400M and the fuselage skin of the Boeing 787 Dreamliner.

The trend towards replacing the aluminium fuselage skin with a carbon fibre reinforced composite, while delivering structural and manufacturing benefits, is likely to have an impact on cabin noise, due to the difference in the sound transmission loss characteristics of the lighter and directionally stiffer carbon fibre matrix composite compared to the structurally equivalent aluminium panel. A specific concern is the vibrational and acoustic response of more composite fuselage designs at the low-frequency end of the audible spectrum, where the traditional aluminium skin was providing an appropriate transmission loss due to its mass.

Therefore, it is of interest to airframe manufacturers to develop a greater understanding of the unsteady pressure field on the cabin outer skin, thereby improving the input data for cabin noise prediction models, in particular, at the low-frequency end of the audible spectrum.

This low-frequency focus is conducive to the use of Implicit Large Eddy Simulations (ILES) for modelling the unsteady jet and the pressure near-field by a direct numerical approach, without having to use either an acoustic analogy or an viscous/inviscid domain splitting technique. In ILES, the large scales of motion are resolved in space and time, while the diffusive effects of the un-resolved sub-grid scale motion on the large scales are accounted for by an appropriate design of the space-time discretization. The large-scale motion, with its associated low-frequency dynamics, is expected to be the main contributor to the lowfrequency components of the unsteady near-field pressure spectrum. It would appear therefore appropriate to neglect in the first instance the contributions from the small-scale high-frequency turbulence, which remain unresolved in ILES.

This direct approach to modelling the unsteady pressure near-field is compatible with the current trend for higher bypass engines, generating jets from larger diameter secondary stream nozzles, that lead to the jet running in greater proximity to the airframe fuselage, with the radial separation distance measured with respect to the nozzle exit diameter. This allows the design of a simulation in which both the jet and the unsteady pressure monitoring points are located in the same computational domain.

*Thermofluids Senior Lecturer, MAIAA.

$\dagger$ Marie Curie Fellow.

${ }^{\ddagger}$ ITN Technical Manager. 
In this paper, the ILES direct approach is tested using the simplified geometry of a single flux cold underexpanded jet from a convergent nozzle. Comparisons are given with time-averaged aerodynamic data from André ${ }^{1}$ to build confidence in the predictions. Numerical flow visualisation is used to identify the known mechanisms of noise radiation from shock-associated noise and screech in the jet plume, with the radiating pressure field visualised by numerical schlieren. Finally, time-resolved ILES predictions are used to probe numerically the near-field pressure at a location that is representative of the outer fuselage geometry from a current civil transport aircraft.

\section{Flow conditions}

Time-dependent simulations are presented of a contoured convergent nozzle with exit diameter $D_{e}=38.0$ $\mathrm{mm}$ and a nozzle exit Mach number $M_{e}=1.0$. The nozzle is operated under-expanded at the stagnation to ambient pressure ratio $p_{s} / p_{\infty}=2.27$. The modelled nozzle lip thickness $t_{e}$ is $0.125 D_{e}$. The flow at the nozzle exit is axial. This cold air jet has an exit stagnation temperature of $288.15 \mathrm{~K}$. The modelled exit and ambient conditions match those in the experimental set-up of André. ${ }^{1}$ The nozzle exit static density, axial velocity, pressure, and temperature are $1.6206 \mathrm{~kg} / \mathrm{m}^{3}, 318.6 \mathrm{~m} / \mathrm{s}, 117.52 \mathrm{kPa}$, and $252.62 \mathrm{~K}$, respectively. The jet discharges in initially quiescent air at temperature $T_{\infty}=288.15 \mathrm{~K}$ and pressure $p_{\infty}=98.0 \mathrm{kPa}$. The Reynolds number, $R e$, based on the jet exit diameter is $1.2 \times 10^{6}$ and the fully expanded jet Mach number $M_{j}=1.15$.

The air flow is modelled under constant specific heat ideal gas assumptions, with specific gas constant $R=287.058 \mathrm{~J} /(\mathrm{kg} \mathrm{K})$ and specific heat ratio $\gamma=1.4$.

Figure 1 gives a schematic of the main flow features that characterise the flow from the nozzle operated at the stated pressure ratio. The velocity difference between the airflow at the nozzle exit plane and the surrounding quiescent air creates a streamwise growing shear layer forming at the nozzle lip. The under-expanded airflow from the nozzle exit plane expands through a fan of expansion waves generated by the surrounding lower ambient pressure. Upon reaching the shear layer, the expansion waves from the nozzle lip refract as compression waves that focus towards the nozzle axis, creating a shock cone, as identified by the arrow in Fig. 1. The jet core, re-compressed by the shock cone, starts a new expansion and this process repeats as a sequence of compressions and expansions. The inflected velocity

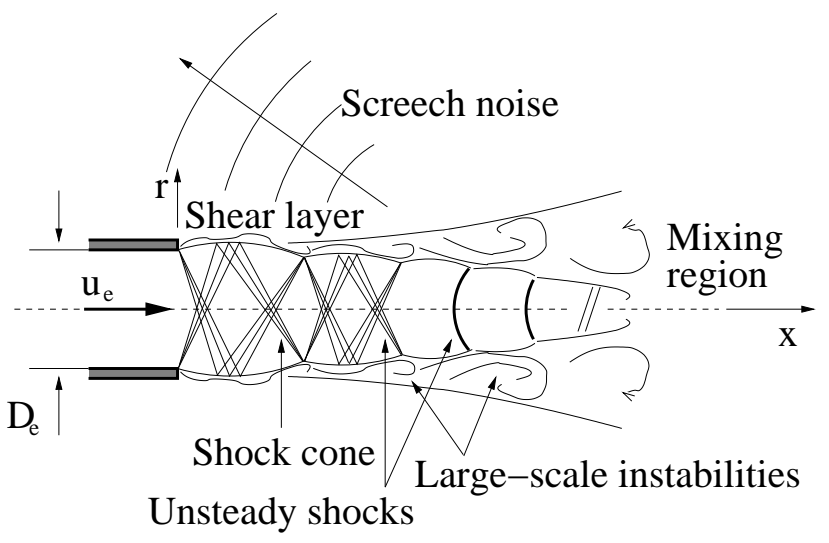

Figure 1. Flow features of an under-expanded jet. profile of the shear layer is receptive to disturbances that convectively amplify, developing into large-scale instabilities, as indicated by arrows in Fig. 1. The resulting shear-layer motion makes the shock-cell structure inside it unsteady. The sketched shock-shear-layer interaction results in screech noise being emitted in the surroundings.

\section{Numerical method}

At the selected flow conditions, the jet under-expansion develops screech. Tam and $\mathrm{Hu}^{2}$ showed that this aero-acoustic phenomenon is mainly an inviscid process, involving the convective amplification of shear-layer instability modes, their interaction with the shock-cell structure, and an intense tonal acoustic feed-back through the subsonic portion of the shear layer to the nozzle lip. The characteristic frequency of this self-excited process is typically tonal and well-defined, prompting the adoption of a modelling approach in which this frequency and its associated large-scale motion are resolved in space and in time. In view of the stated focus on resolving the lower-frequency unsteady pressure field, the higher-frequency motion mainly associated to the small-scale turbulence is not resolved. A space filtering criterion can then be introduced through the flow governing equations, the compressible Navier-Stokes equations, by which these are tophat filtered by the unit-cell volume $\Delta$ of the computational mesh. In the filtered equations, each scalar variable $f$ is represented by the summation of its top-hat filtered value, $\langle f\rangle$, plus a fluctuation $f^{\prime \prime}$, so that 
$f=<f>+f^{\prime \prime}$. Upon neglecting product terms involving the small-scale density fluctuations, the resulting equations are formally identical to the Reynolds Averaged Navier-Stokes (RANS) equations. In Large Eddy Simulations (LES), the products of the fluctuating quantities are modelled based on the filtered quantities, to provide turbulence closure. In Implicit Large Eddy Simulations, the effects of the fluctuating quantities on the filtered quantities are taken as mainly diffusive and dissipative and the numerical dissipation and diffusion in the numerical scheme is relied upon to model such effects. By this approach, all products of fluctuating quantities $<f^{\prime \prime} f^{\prime \prime}>$ in the filtered equations are set to zero, thereby reducing the computational effort compared to a Large Eddy Simulation.

By space filtering the Navier-Stokes equations in cylindrical coordinates $(x, r, \theta)$, the axi-symmetric flow governing equations become

$$
\frac{\partial \mathbf{U}}{\partial t}+\frac{\partial \mathbf{F}}{\partial x}+\frac{\partial r \mathbf{G}}{r \partial r}=\frac{\partial \mathbf{F}_{v}}{\partial x}+\frac{\partial r \mathbf{G}_{v}}{r \partial r},
$$

where the vector of the conservative variables $\mathbf{U}$ and the inviscid $(\mathbf{F}, \mathbf{G})$ and viscous $\left(\mathbf{F}_{v}, \mathbf{G}_{v},\right)$ flux vectors are

$$
\begin{aligned}
\mathbf{U} & =\left(\rho, \rho u_{x}, \rho u_{r}, \rho e_{s}\right)^{T}, \\
\mathbf{F} & =\left(\rho u_{x}, \rho u_{x}^{2}+p, \rho u_{x} u_{r}, \rho u_{x} h_{s}\right)^{T}, \\
\mathbf{G} & =\left(\rho u_{r}, \rho u_{r} u_{x}, \rho u_{r}^{2}+p, \rho u_{r} h_{s}\right)^{T}, \\
\mathbf{F}_{v} & =\left(0, \tau_{x x}, \tau_{x r}, u_{x} \tau_{x x}+u_{r} \tau_{x r}+q_{x}\right)^{T}, \\
\mathbf{G}_{v} & =\left(0, \tau_{r x}, \tau_{r r}, u_{x} \tau_{r x}+u_{r} \tau_{r r}+q_{r}\right)^{T} .
\end{aligned}
$$

Equation (1) is stated in terms of space-filtered normalised variables with respect to the nozzle exit plane conditions $\left(\rho_{e}, u_{e}, T_{e}, M_{e}\right)$. Lengths are normalised by the nozzle exit diameter $D_{e}$, density $\rho$ by $\rho_{e}$, velocities $\left(u_{x}, u_{r}\right)$ by $u_{e}$, pressure $p$ by $\rho_{e} u_{e}^{2}$, specific stagnation enthalpy $h_{s}$ and specific stagnation energy $e_{s}$ by $u_{e}^{2}$, temperature $T$ by $T_{e}$, time $t$ by $D_{e} / u_{e}$, and molecular viscosity $\mu$ by $\mu_{e}$. All variables in Eq. (2) are space-filtered quantities and the symbol $\langle>$ is omitted for conciseness, as in the remainder of this paper.

The ideal gas state equation $p=\rho T /\left(\gamma M_{e}^{2}\right)$ relates $p, \rho$, and $T$. The viscous stress tensor $\tau$ is

$$
\begin{aligned}
\tau_{x x} & =2 \frac{\mu}{R e} \frac{\partial u_{x}}{\partial x}-\frac{2}{3} \frac{\mu}{R e}\left(\frac{\partial u_{x}}{\partial x}+\frac{\partial r u_{r}}{r \partial r}\right) \\
\tau_{r r} & =2 \frac{\mu}{R e} \frac{\partial u_{r}}{\partial r}-\frac{2}{3} \frac{\mu}{R e}\left(\frac{\partial u_{x}}{\partial x}+\frac{\partial r u_{r}}{r \partial r}\right) \\
\tau_{x r} & =\tau_{r x}=\frac{\mu}{R e}\left(\frac{\partial u_{x}}{\partial r}+\frac{\partial u_{r}}{\partial x}\right)
\end{aligned}
$$

and the heat flux vector is

$$
\begin{aligned}
q_{x} & =\frac{1}{(\gamma-1) M_{e}^{2} \operatorname{Re}} \frac{\mu}{\operatorname{Pr}} \frac{\partial T}{\partial x} \\
q_{r} & =\frac{1}{(\gamma-1) M_{e}^{2} \operatorname{Re}} \frac{\mu}{\operatorname{Pr}} \frac{\partial T}{\partial r}
\end{aligned}
$$

where $\operatorname{Pr}=0.72$ is the Prandtl number. The molecular viscosity $\mu$ is estimated by the Sutherland law

$$
\mu=T^{3 / 2}\left(1+110 / T_{e}\right) /\left(T+110 / T_{e}\right)
$$

The specific stagnation energy $e_{s}$ and the specific stagnation enthalpy $h_{s}$ are given by

$$
\begin{aligned}
e_{s} & =T /\left[\gamma(\gamma-1) M_{e}^{2}\right]+\left(u_{x}^{2}+u_{r}^{2}\right) / 2, \\
h_{s} & =e_{s}+p / \rho .
\end{aligned}
$$

The finite-volume discrete form of the flow governing equations, Eq. (1), is applied to the jet computational domain of Fig. 2, where only the top half of the meridional plane through the jet axis is shown for clarity. The computational domain extends $6 D_{e}$ radially and $20.5 D_{e}$ axially. The meridional plane is discretised by a uniform rectangular mesh of $1312 \times 384$ cells, giving a constant unit-cell size $(\Delta x, \Delta r)$ of 


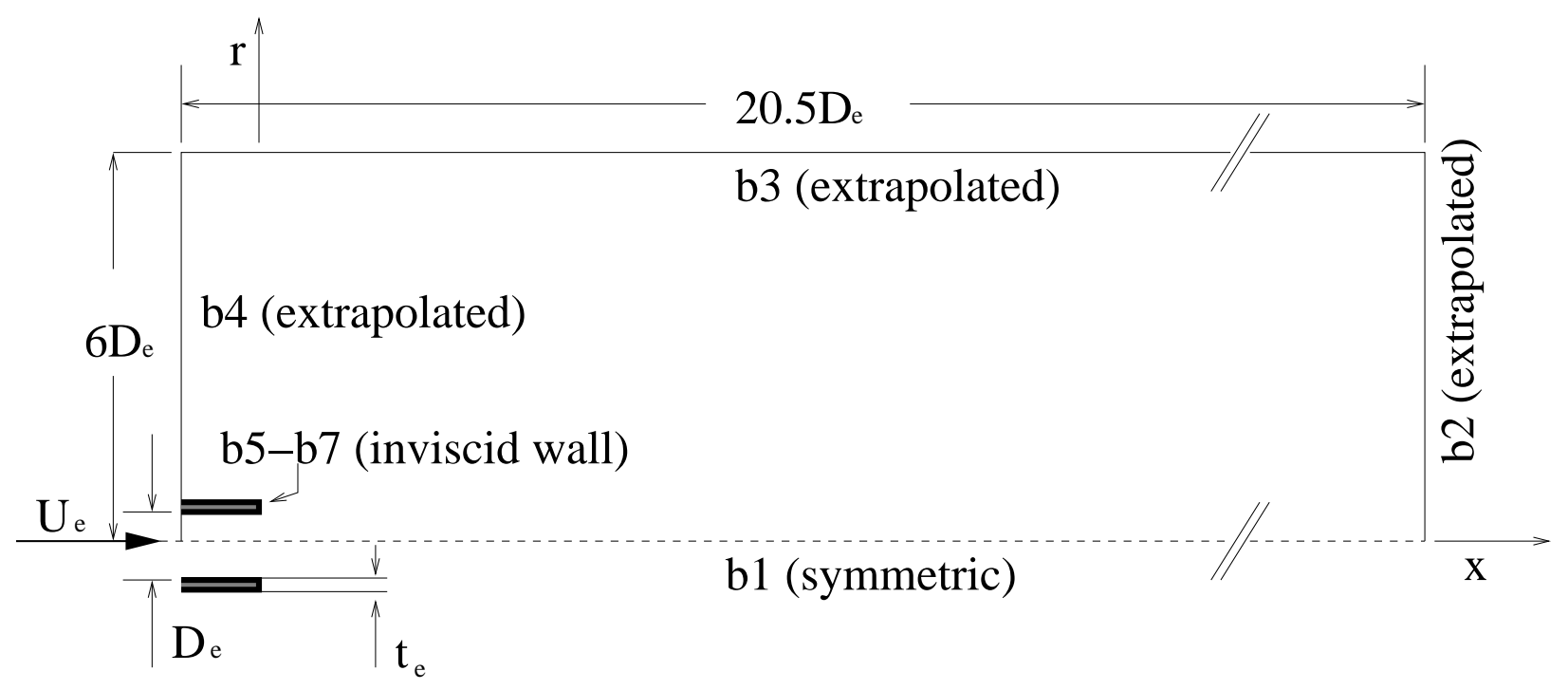

Figure 2. Computational domain and boundary conditions.

$\left(0.0157 D_{e}, 0.0156 D_{e}\right)$ on the $(x, r)$ plane. Adopting a uniform spatial discretisation in this plane has the advantage that, for axi-symmetric flows, the same spatial filter $\Delta$ is applied consistently to the governing equations, resulting in a uniform filtering effect with a constant cut-off wavenumber in Fourier space. This is the baseline mesh used for obtaining most of the numerical predictions reported in this paper.

The Implicit Large Eddy Simulation approach relies on selecting an appropriate discretization in both space and time to model the dissipative effects of the unresolved sub-grid structures on the resolved scales of motion. The sensitivity of the predictions on the spatial discretisation is therefore evaluated by considering a uniformly refined rectangular mesh of $1968 \times 576$ cells, with a constant unit-cell size $(\Delta x, \Delta r)$ of $\left(0.0105 D_{e}, 0.0104 D_{e}\right)$. The mesh refinement ratio 1.5 in both the $x$ and $r$ directions gives a baseline to refined mesh size ratio of 2.25 .

The jet plume is characterised by compressible flow features, such as shocks, expansion fans, and regions of significant flow shear. These features produce significant localised gradients in the conservative variables. To resolve these features, a time-marching Total Variation Diminishing (TVD) scheme is used to integrate the space-filtered flow governing equations in finite-volume form. The inviscid fluxes at the computational cell interfaces are evaluated using the second-order form of the $\mathrm{Roe}^{3,4}$ flux difference approximate Riemann solver. Numerical stability is provided by the min-mod flux limiter. Second-order central differences are used to evaluate the viscous fluxes at the cell interfaces.

The computation is time-marched by a two-step Runge-Kutta scheme implemented in the low storage form of $\mathrm{Hu}$ et al. ${ }^{5}$ The standard Runge-Kutta coefficients $(1.0,0.5)$ are used for numerical stability. The numerical integration procedure is further described in Rona and Zhang. ${ }^{6}$

Conventional computational fluid dynamic boundary conditions are used, as shown in Fig. 2. A reflecting boundary b1 is imposed along the jet axis where $\mathbf{U}_{i,-j, k}=\mathbf{U}_{i, j, k}^{*}$ for $j=1$ and $j=2$, where $r=j \Delta r$ and $\mathbf{U}^{*}=\left(\rho, \rho u_{x}-\rho u_{x} \Delta i, \rho u_{r}-\rho u_{r} \Delta j, \rho e_{s}\right)^{T}$. The open flow boundaries b2-b4 are treated by linear extrapolation of the flow state from the computational domain interior. The solid boundaries b5-b7 are modelled by the wall condition $r \mathbf{U}_{i,-j, k}=r \mathbf{U}_{i, j, k}^{*}$ for $j=1$ and $j=2$ and $\mathbf{U}_{-i, j, k}=\mathbf{U}_{i, j, k}^{*}$ for $i=1$ and $i=2$ for the horizontal and vertical surfaces, respectively. At the nozzle inlet, the flow state $\left(\rho_{e}, u_{e}, T_{e}, M_{e}\right)$ is imposed constant throughout the computation.

At the start of the computation, the entire computational domain is primed with the ambient zero flow conditions and the jet is impulsively started by the nozzle inlet supersonic inflow. This abrupt start condition challenges the computational stability of the scheme, as it generates a downstream propagating bow shock outside the nozzle. The flow is therefore time-marched by a fixed Courant number of 0.8 to the non-dimensional time $t=65$ to obtain a stationary flow, as indicated by the static pressure monitored at $x=1.5 D_{e}$ and $x=2.5 D_{e}$ on the jet axis. By $t=65$, the bow shock has propagated through the downstream boundary b2 and a jet with an organised shock-cell pattern is developed. 
During this initial transient, screech develops in the axi-symmetric flow without forcing. The jet selfselects its screech frequency $f$ and Strouhal number $S t r=f D_{e} / u_{e}$. Specifically, Str $=0.7$. From $t=65$, the computation is further advanced to the computational time $t=586$ at the fixed time step of $\Delta t=4.34 \times 10^{-3}$ on the baseline mesh, which corresponds to a Courant number of approximately 0.56 , based on the nozzle outflow conditions. On the refined mesh, after the initial transient, the computation is advanced by a fixed time step of $\Delta t=2.47 \times 10^{-3}$, which corresponds to a Courant number of approximately 0.476. Flow statistics are collected by a running average of the time-dependent simulations after the end of the initial transient.

\section{Results}

\section{A. Time-averaged flow predictions compared to experiment}

The time-dependent ILES predictions obtained from the baseline and refined computational meshes do not converge to a statistically stationary flow. Specifically, the flow unsteadiness evolves over time, exhibiting modes that alternate in the pressure spectrum over time, as discussed in section C later on. A quasistationary flow regime establishes, in which the flow dynamics is disrupted by intermittent vortex pairing, which manifests as a seemingly stochastic occurrence. These random occurrences affect the time-averaged flow statistics. In this paper, the time-averaged statistics are interpreted as the ones associated to selfsustained steady screech. Therefore, a short time average of the jet flow was computed by averaging over 30 full periods of the fundamental screech frequency on the baseline mesh and 100 full periods of the fundamental screech frequency on the refined mesh, as determined from monitoring the pressure on the jet axis at $x=1.5 D_{e}$.

Figure 3 shows the resulting time-averaged Mach number $(M)$ distribution along the jet axis. The numerical prediction from the baseline mesh ILES, shown by the continuous line, and the prediction from the refined mesh ILES, shown by the dash-dot line $(-\cdot-)$, are compared with measurements by André, ${ }^{1}$ denoted by open circles ( $\circ$ ). Dashed line segments $(--)$ connect the experimental points to highlight the trend in the measurements.

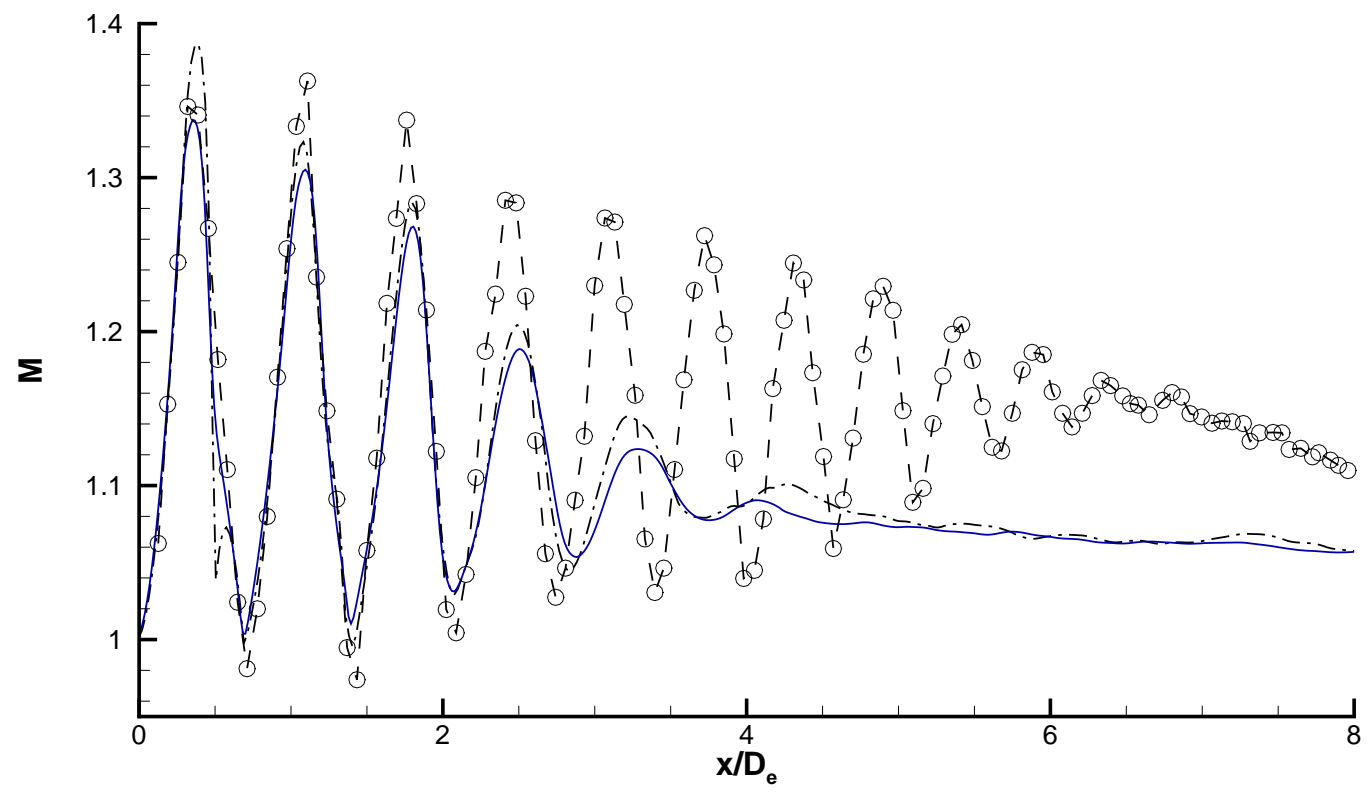

Figure 3. Time-averaged Mach number distribution along the jet axis. (-) baseline mesh ILES averaged over $30 T,(-\cdot-)$ refined mesh ILES averaged over $100 T$ and $(\circ)$ experiment. $^{1}$

The numerical predictions show an oscillatory axial Mach number distribution with the oscillation ampli- 
tude monotonically decreasing with increasing axial distance from the nozzle exit plane. This trend indicates a monotonic decrement in the intensity of the compression and expansion waves with increasing axial distance, which is consistent with the monotonic loss of momentum from the jet core to the surrounding air that generates a subsonic jet plume further downstream. The experimental results show that the first Mach number maximum, located at $x \sim 0.4 D_{e}$, is lower than the subsequent maximum at $x \sim 1.2 D_{e}$, deviating from this trend. This discrepancy is perhaps due to measurement interference effects with the nozzle lip that may have limited the local flow expansion in the experiment. Notwithstanding this effect, the numerical predictions from the baseline and the refined mesh display a good overlap with the measurements in Figure 3 up to the first Mach number maximum, where the numerical predictions lie either side of the measurement. This indicates that the amplitude and the location of the Prandtl-Mayer expansion wave at the nozzle lip is well predicted by just using an axi-symmetric ILES computation. The good overlap continues up to the first Mach number minimum, which denotes the effect of the first conical shock in the shock-cell system. The intensity and location of the flow recompression is well predicted on both the baseline and the refined meshes. Due to the flow acceleration through the nozzle in experiment, ${ }^{1}$ the nozzle exit velocity profile is nearly a top-hat and the exit boundary layer is thin both in experiment and in the simulation so that the first expansion and conical shock can be regarded essentially as inviscid flow features. The good match between experiment and predictions shows the good shock capturing abilities of the approximate Riemann solver implemented in the ILES method. The refined mesh prediction displays a secondary expansion at $x \sim 0.5 D_{e}$ and a similar feature has been reported from independent LES simulations at CERFACS. ${ }^{7}$ Further downstream, while the spacing between subsequent Mach number maxima and minima is in broad agreement between prediction and experiment, the amplitude of the streamwise Mach number fluctuations is lower in the predictions compared to the experiment. This is likely to result from the axial precession of the shock cells, due to the shear-layer motion. The movement of the shock cells during the running average causes shock smearing in the time-averaged flow, reducing the amplitude of the extrema in the axial Mach number distribution. As the shear layer motion grows in amplitude with increasing downstream distance from the nozzle exit plane, the shock cells further away from the nozzle exit are more affected by this shock smearing process in the time-averaged flow, as indicated in Figure 3. The time-averaged Mach number distribution from the baseline mesh exhibits maxima and minima with the same monotonic decreasing trend as the refined mesh results. There is a good agreement in the location of the extrema up to the last expansion wave, where the Mach number maximum from the refined mesh is predicted further downstream. The amplitude of the Mach number extrema from the refined mesh is higher than from the baseline mesh, due to the reduction in the numerical dissipation provided by the greater spatial resolution. Whereas the ILES method is not designed to provide strictly mesh-independent results, due to the lack of an explicit turbulence closure model, the current convergence between the baseline mesh and the refined mesh predictions gives confidence that the main time-mean flow geometry is adequately modelled.

Table 1. Axial length of shock cells.

\begin{tabular}{lcc}
\hline Shock spacing & Baseline mesh prediction & Measurement $^{1}$ \\
\hline$L_{1} / D_{e}$ & 0.72 & 0.73 \\
$L_{2} / D_{e}$ & 0.73 & 0.68 \\
$L_{3} / D_{e}$ & 0.69 & 0.68 \\
$\bar{L} / D_{e}$ & 0.71 & 0.70 \\
\hline
\end{tabular}

Table 1 shows the comparison between the shock cell lengths predicted by the ILES using the baseline mesh and the ones computed from the measurements. ${ }^{1}$ Both measurements and predictions indicate a monotonic reduction in shock cell length with axial distance. The shear layer grows in the downstream direction by the entrainment of the low speed flow that surrounds it. Axial momentum transfer to the entrained fluid from the high-speed flow closer to the jet axis reduces the enclosed high-speed flow radius. The boundary between the shear layer and the high-speed flow is therefore convex, as sketched in Fig. 1, and the axial velocity progressively reduces, due to the irreversible axial momentum loss through the shocks. This results in progressively more normal compression and expansion waves, which reduce the shock spacing in the axial direction from $L_{1} / D_{e}$ in the first shock cell to $L_{3} / D_{e}$ in the third shock cell.

The predicted average shock spacing $\bar{L}$ is in broad agreement with experiment over the first three shock cells. Figure 3 indicates that the agreement in the shock spacing progressively decreases with increasing 
shock-cell number. The shear layer thickness is smallest over the first three shock cells, increasing monotonically in the positive axial direction. The time-averaged shear layer thickness is determined by the diffusive and dissipative effects on the flow momentum. In the Implicit Large Eddy Simulation, these effects are numerical scheme dependent, therefore further improvements in the numerical mesh and in the limiter function are likely to improve the current flow predictions.

\section{B. Time-resolved flow predictions}

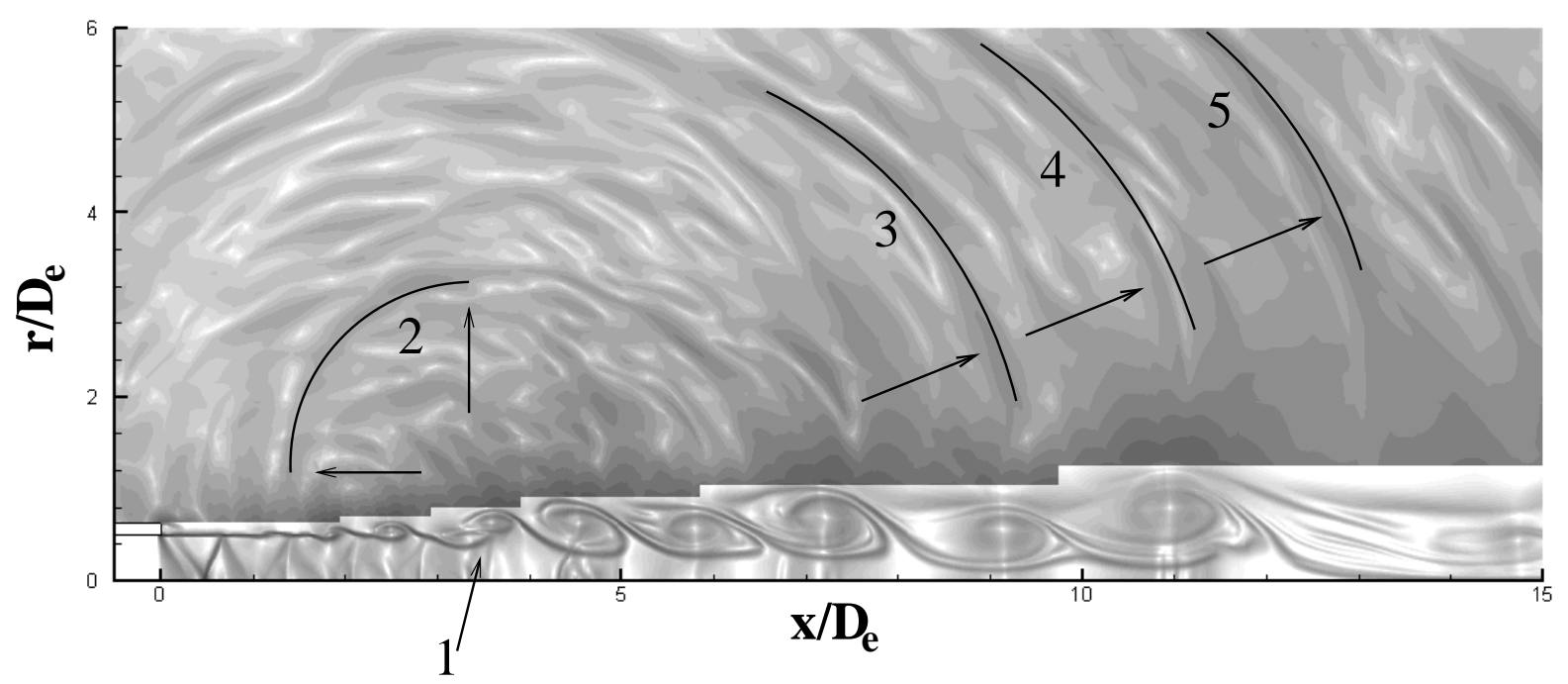

(a) Non-dimensional time $\tau=586$.

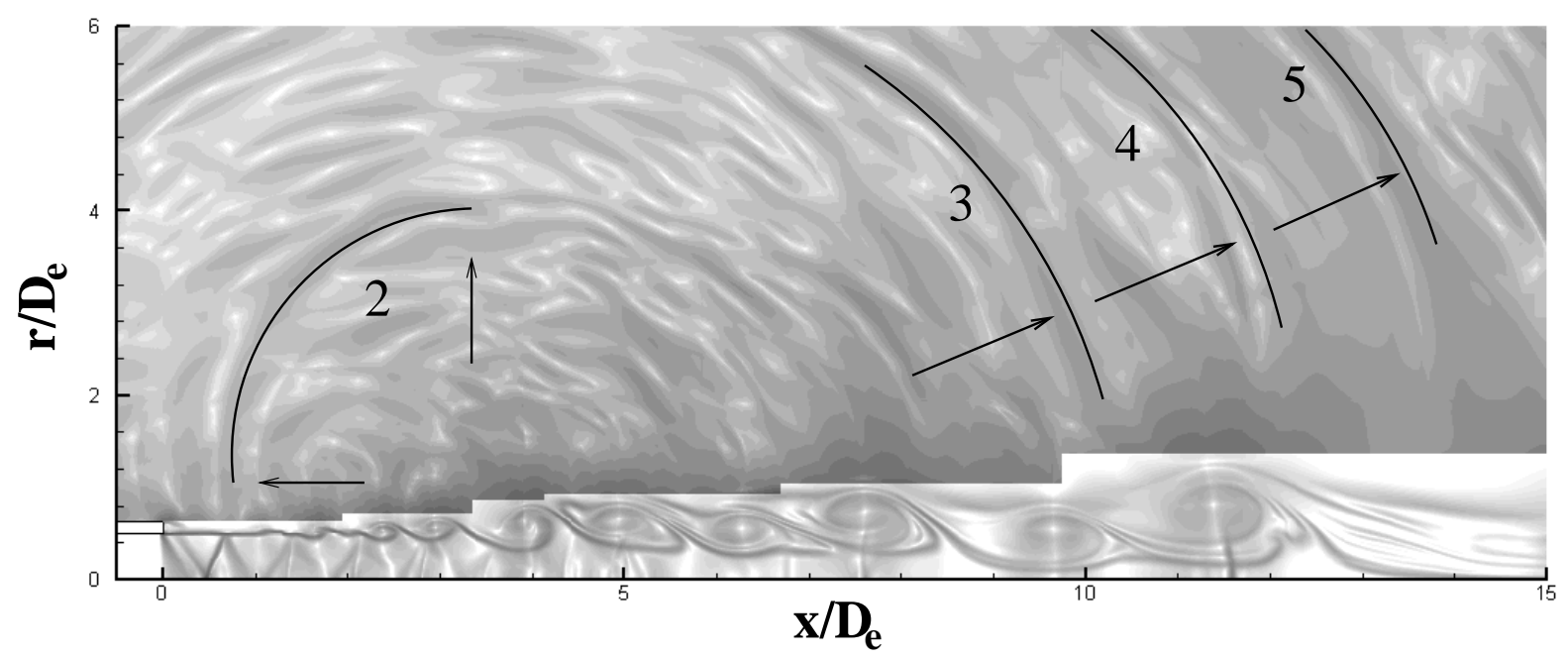

(b) Non-dimensional time $\tau=587$.

Figure 4. Pseudo-schlieren visualisation of the jet at non-dimensional times $(a) \tau=586$ and $(b) \tau=587$.

Figure 4 shows two snapshots from the time-dependent ILES that are approximately spaced in time by half a fundamental period of screech. This time-sequence of flow visualisations is obtained from the commercial post-processor Tecplot 360 using a pseudo-schlieren technique. Specifically, the density gradients in the flow are visualised by plotting the scalar $\phi=|\partial \rho / \partial x|+|\partial \rho / \partial r|$. This scalar is broadly similar to using a circular knife-edge, or a mechanical optical stop of small aperture as knife-edge, in experiment. As the density gradients in the shock-cell structure inside the shear layer are higher than the ones in the acoustically perturbed flow that surrounds the jet plume, the computational domain has been divided in two zones, for visualization purposes. In both zones, iso-levels of $\phi$ are shown using an exponential distribution. The inner region, close to the jet axis, uses a $\phi$ scale in which the minimum is $10^{3}$ times the one in the outer region. 
Large-scale shear-layer oscillations characterise the numerical predictions of this under-expanded jet. These oscillations affect the compressible flow features in the high-speed flow bounded by the shear layer. The shear-layer oscillations originate in the proximity of the nozzle lip and amplify in the positive axial direction by convective amplification. The shear-layer instability is a toroidal, or varicose, mode. This mode is determined by the axi-symmetry imposed by the flow solver. At the selected flow conditions, André ${ }^{1}$ reports that the dominant screech mode frequencies recorded in experiment are from A1 and A2 modes, which are axi-symmetric screech modes. In reviewing jet screech, Raman ${ }^{8,9}$ reports experimental evidence indicating that axi-symmetric screech modes are typically encountered at modest degrees of under- or overexpansion. In the present investigation, flow predictions are obtained for the jet from a Mach 1 nozzle operated at the fully expanded jet Mach number $M_{j}=1.15$. At this modest degree of under-expansion, the axi-symmetric modes reported by André ${ }^{1}$ are therefore in line with the established literature. It is possible that secondary helical or flapping shear-layer instabilities could have been present in experiment in addition to axi-symmetric modes. These additional modes are not represented in the axi-symmetric results shown herein and a computationally more expensive three-dimensional flow simulation would be required to resolve them.

Some interesting insight can be gained from the analysis of these axi-symmetric results. Between the nozzle exit plane and $x=2 D_{e}$, the shear layer displays radial oscillations of modest amplitude. These oscillations are likely to force the natural modes of the axi-symmetric shear layer that amplify with convection. Over the range $2 D_{e}<x \leq 5 D_{e}$, the instability modes grow to a finite-amplitude, developing non-linearities that cause the shear layer to roll-up. A rolled-up shear-layer structure is indicated by the arrow 1 in Figure 4(a). A similar shear-layer evolution was observed in the axi-symmetric shear layer experiments by Gharib and Roshko, ${ }^{10}$ where the convective amplification of the instability modes indicates a KelvinHelmholtz type instability.

The non-linear development of the downstream propagating shear-layer instabilities limits their growth by mode saturation. The rolled-up shear layer interacts with the quasi-periodic shock-cell pattern, generating an unsteady pressure field. This provides upstream feed-back at the nozzle lip for the excitation of new shearlayer disturbances, through the shear-layer receptivity, establishing a closed-loop instability mechanism. The match in wavelength between the standing pressure wave pattern and the shear-layer convective instabilities locks the flow in a self-sustained tonal instability, with the frequency determined by the propagating speeds of the upstream travelling pressure waves and of the downstream convecting shear-layer instabilities. This selects the jet screech frequency.

The level of spatial resolution in the baseline mesh does not allow rendering the detail of how, by interaction with the shock-cell pattern, the toroidal shear layer instabilities generate a propagating pressure field outside the jet. This detail is provided independently by numerical simulations by Manning and Lele, ${ }^{11}$ where numerical simulations of a vortex sheet past an oblique shock, modelled as a finite-thickness compression wave, is presented. In Manning and Lele, ${ }^{11}$ the vortex sheet is artificially perturbed by a spatial pattern of downstream convecting vortices. These simulations show that outer pressure waves are generated by the shock leaking through the shear layer, as a vortex passes over the shock tip. As the pressure waves are released in the subsonic outer flow, they propagate at the local speed of sound.

The frequency of emission of the pressure waves at the shock-shear layer interactions is that of their source, that is, the screech frequency. As the speed of propagation is the speed of sound, this generates pressure waves of a relatively long wavelength that can be resolved by the current level of space discretization of the baseline mesh.

The wave pattern outside the jet plume outlined in Figure 4 shows the wave fronts generated by screech. An upstream propagating wave system, labelled as 2 in Figures 4(a) and 4(b), is shown by a continuous arc. This wave progresses towards the nozzle lip in the time sequence between Figures 4(a) and 4(b). The radius of the arc indicates that this wave originates in the shear layer at about $x \sim 3 D_{e}$. This wave is likely to be the signature of the upstream feed-back screech pressure wave component released through the shear-layer envelope. Downstream propagating waves are shown in Figures 4(a) and 4(b) by labels 3, 4 and 5. These wave fronts are shown to move by approximately half the distance between two consecutive fronts between the two snapshots, which is consistent with these waves being generated from perturbations at the fundamental screech frequency. The arc shape of these wave fronts defines a radius of propagation the origin of which can be located in the jet shear layer over the range $3 D_{e} \leq x \leq 4 D_{e}$.

The numerical schlieren visualisations of Figure 4 display a shear layer in which a train of regularly spaced self-similar vortices grow in the positive axial direction. The flow strain regions between consecutive 


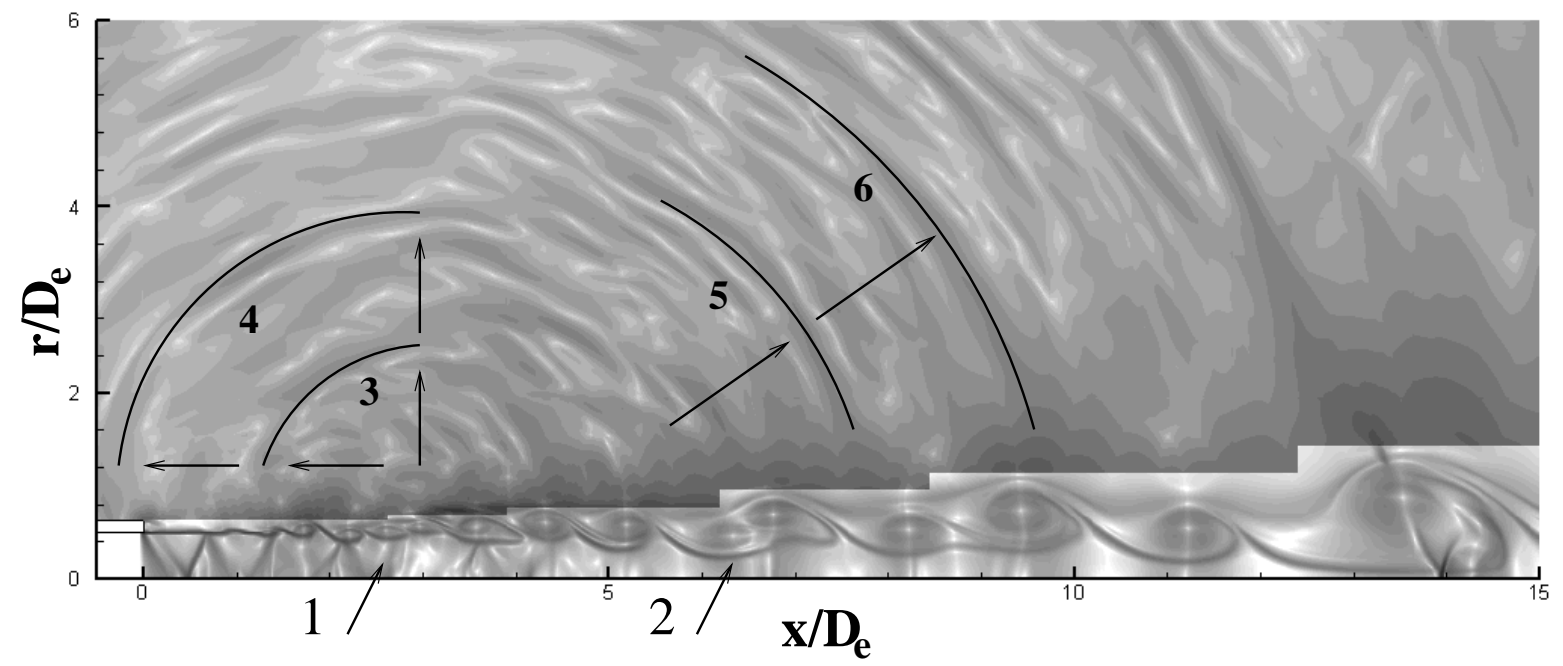

(a) Baseline mesh showing vortex pairing.

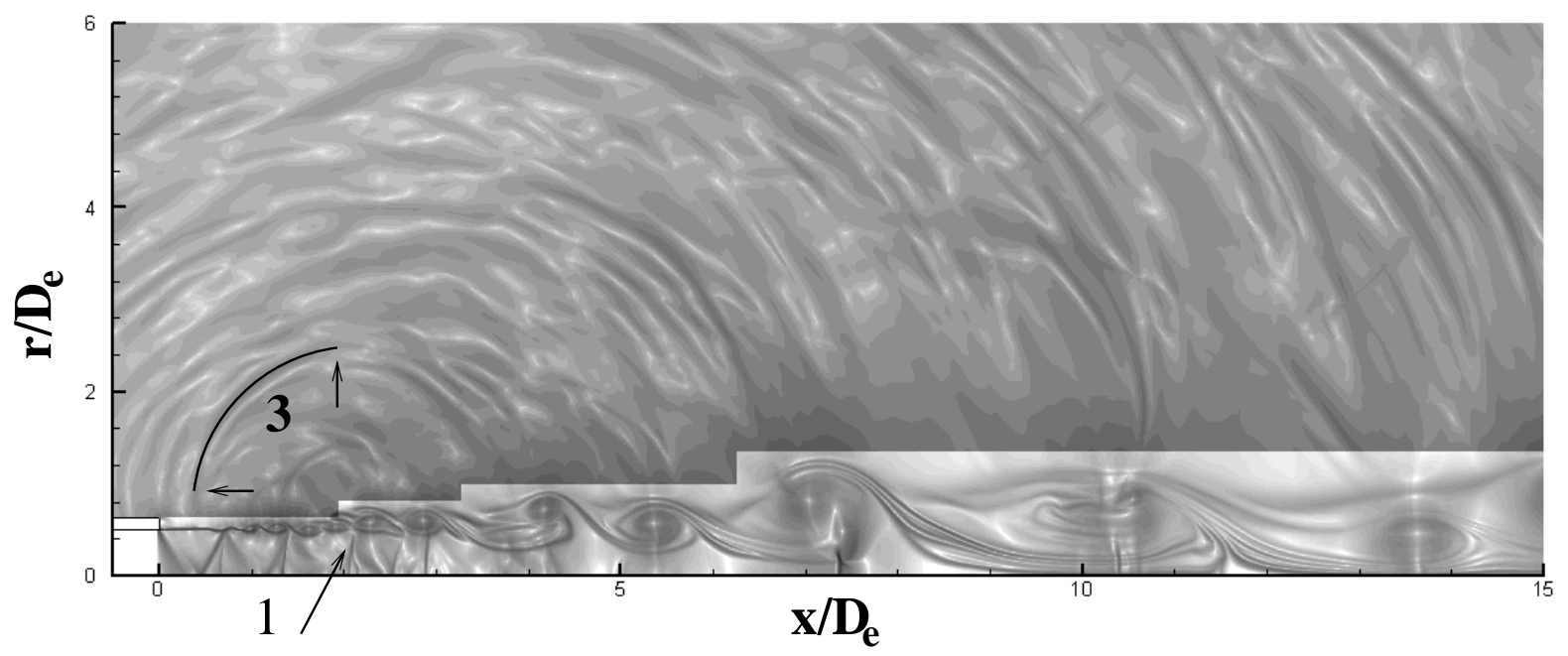

(b) Refined mesh showing vortex pairing.

Figure 5. Numerical schlieren visualisation. (a) $1312 \times 384$ baseline mesh, (b) $1968 \times 576$ refined mesh. 
vortices are topologically similar to one another. This makes this flow regime amenable to statistical analysis for determining the mean rate of mixing of the jet and other time-averaged flow kinetic properties. At the selected test conditions, this orderly flow regime is not stationary and it is intercalated by a flow characterised by a greater interaction among the vortices. An example of this intermittent vortex interaction is given in Figure 5(a), where iso-levels of the normalised density gradient $\phi$ are shown on the same scale as in Figure 4(a). Over the range $2 D_{e} \leq x \leq 3 D_{e}$, three vortices are shown interfering with one another along the shear layer, where indicated by arrow 1 . Further downstream, over the range $6 D_{e} \leq x \leq 7 D_{e}$, the numerical pseudo-schlieren visualisation indicates that vortex pairing is taking place where indicated by arrow 2 . These non-linear shear layer interactions are likely to be responsible for stochastic variations in the aerodynamic pressure spectrum over time, which are discussed in section C later on.

Figure 5(b) is a selected snapshot from the time-dependent predictions with the $1968 \times 576$ refined computational mesh. The flow is visualised using the same pseudo-schlieren technique as Figures 4 (a) and 5 (a) using iso-levels of $\phi$ with an exponential distribution. Figure 5(b) displays a broad similarity in the wave pattern outside the jet plume compared to Figure 4(a). An upstream propagating wave system is shown over the range $0 \leq x \leq 2 D_{e}$, with an arc shaped wave front located approximately at $x=0.5 D_{e}$ outside the shear layer. The radius of the wave front arc indicates that the origin of this perturbation is at $x \sim 2 D_{e}$ in the jet shear layer. This is a more upstream location with respect to the corresponding feature in Figure 4(a) and is likely to result from the vortex pairing event taking place at this axial location, as shown by arrow 1. Figures $5(\mathrm{a})$ and 5 (b) indicate that vortex pairing has a significant transient effect in the location of the upstream propagating pressure waves and it is therefore likely to affect the stationarity of screech. Such effect is captured on both the baseline and the refined computational meshes, giving some confidence in the choice of the spatial discretization level used in these Implicit Large Eddy Simulations.

It is encouraging to notice that the computational domain, at this computational time, appears not to display strong pressure wave reflections from the through-flow boundaries b2-b4. This welcomed result was relatively unexpected, given the intense on-going research on acoustically non-reflecting boundary conditions for aeroacoustic computations. A number of concurrent effects are thought to be in action for producing this result. The long wavelength of the radiating pressure field is most likely within the well-resolved wavenumber range of the second-order numerical scheme, ${ }^{12}$ while any high-wavenumber reflection from the outflow boundaries is likely to be aggressively damped by the min-mod limiter. The total time covered by the computation, $0 \leq t \leq 587$, is relatively short, preventing the amplitude of standing waves from building up to a level that is comparable to that of the screech generated pressure fluctuations. Screech is a high-amplitude tone in the radiating noise spectrum and is likely to remain detectable over a background of otherwise significant numerical noise.

\section{Near-field pressure}

It is of interest to explore the unsteady aerodynamic pressure predictions at locations that are representative of the fuselage outer skin of a commercial transport aircraft, as a preliminary exercise to determine the viability of a direct approach for modelling the outer skin aerodynamic pressure forcing due to the jet flow at cruise conditions.

At this stage, the outcome of this investigation is just whether such a direct modelling approach is feasible, since there are several significant differences between the jet configuration of this numerical experiment and that from a commercial turbofan engine. In fact, the engine jet is typically dual flux, using an annular nozzle configuration run at a modest degree of under-expansion to achieve a high propulsive efficiency at cruise.

Estimates of the outer fuselage unsteady pressure are obtained by scaling the jet geometry based on the bypass flow nozzle exit outer diameter and monitoring the pressure at two positions corresponding to the mid-cabin outer fuselage. Installation effects from the wing and the fuselage on the jet and on the acoustic near-field are not included in this model and the jet axis is assumed parallel to the fuselage skin. Figure 6 shows the resulting geometry, scaled by $D_{e}$, based on an approximate plan view of the BAe 146 Series 200 aircraft. ${ }^{13}$ The mid-cabin fuselage outer skin lies along a line $A B$, with $A=\left(1.5 D_{e}, 3 D_{e}\right)$ and $B=\left(2.5 D_{e}, 3 D_{e}\right)$ in the $(x, r)$ cylindrical reference system of Fig. 1 . The line $A B$ lies well within the computational domain of the simulation, clear from the computational domain boundaries. This supports the direct modelling approach in the computation of the local pressure field.

Figure 7 shows the short-time spectra of aerodynamic pressure predicted at $A$ and $B$ together with the short-time spectra predicted at the same axial locations along the jet shear layer, at $r=0.5 D_{e}$, and along the jet axis, at $r=0$. The axes of the short-time spectra are made dimensional for consistency with 


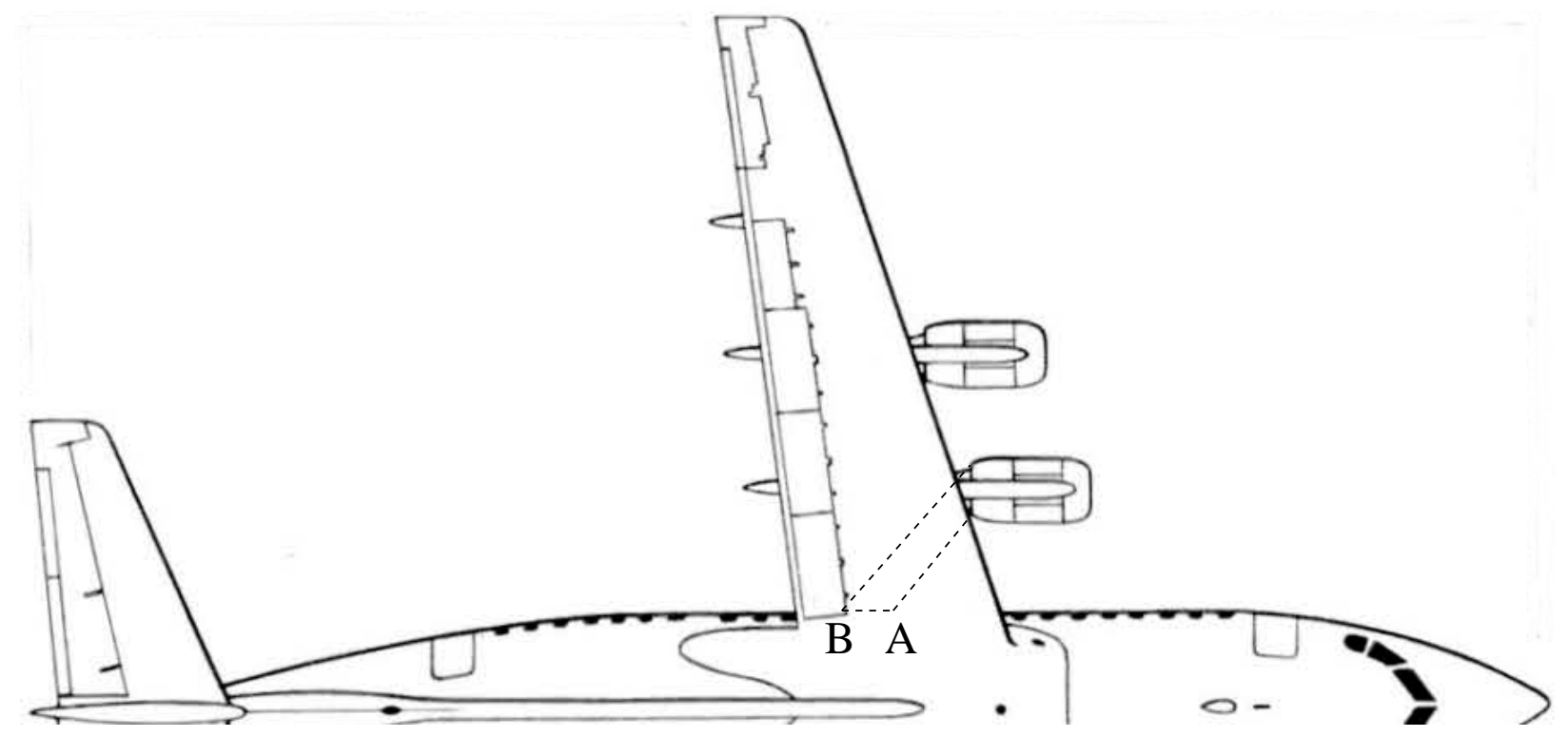

Figure 6. Plan view of civil transport aircraft showing the cabin position relative to the inboard engine bypass nozzle. Plan view adapted from 'Jane's all the world's aircraft'. ${ }^{13}$

the short-time spectra of aerodynamic pressure measured by André ${ }^{1}$ The short-time spectra indicate the presence of tones the amplitude of which varies over time. Over the computed flow time history, there is no obvious regular pattern to this variation, with individual tones becoming absent over both short and long time windows. This points to an intermittent behaviour of the axi-symmetric flow.

The experimental investigation of André ${ }^{1}$ reported A1 and A2 type screech tones at $5171 \mathrm{~Hz}$ and at 5841 $\mathrm{Hz}$ at the same nozzle pressure ratio as the present investigation. These tones are likely to be related to competing instabilities, as they appear to alternate in intensity in the measured short-time spectra. ${ }^{1}$ In the current predictions, Figures 7(c) and 7(d) show a tone at $5834 \mathrm{~Hz}$ that is sustained over time and a more intermittent tone at the lower frequency of $4891 \mathrm{~Hz}$ inside the jet shear layer. The competing behaviour between these modes is less evident in the predictions than in experiment. A third more intense tone is predicted at $13360 \mathrm{~Hz}$ at the position closest to the nozzle exit plane in Figure 7(c). This tone is persistent over time and it is much reduced in amplitude further downstream, in Figure 7(d). Along the jet axis, the frequencies corresponding to the A1 and A2 modes are dominant over several of the time segments reported in Figures 7(a) and 7(b), with the $13360 \mathrm{~Hz}$ higher frequency tone being of lower amplitude compared to the shear-layer monitoring locations of Figures 7(c) and 7(d). This trend is confirmed in the short-time spectra outside the jet shear layer shown in Figures 7(e) and 7(f). The $13360 \mathrm{~Hz}$ higher frequency tone is probably associated with incompressible or 'hydrodynamic' pressure fluctuations due to the convecting vorticity in the shear layer. These are not radiating pressure fluctuations and they decay faster with distance from the shear layer than the acoustic emissions of screech. The significant reduction in amplitude of these high frequency pressure fluctuations at $x=2.5 D_{e}$ compared to $x=1.5 D_{e}$ at $r=0.5 D_{e}$ is consistent with the replacement of small scale instabilities by larger scale instabilities in the growing shear layer.

\section{Conclusions}

A time-resolved numerical scheme is presented for modelling the flow from a sonic nozzle run incorrectly expanded. The method has been tested on a jet from a contoured convergent nozzle operated underexpanded, featuring screech. In a flow characterised by large-scale instabilities of mainly inviscid nature, such as screech, the Implicit Large Eddy Simulation (ILES) approach is shown to provide valuable insight in the time-dependent fluid mechanics. The absence of explicit turbulence closure in ILES creates difficulties in predicting the jet shear layer growth rate and requires greater attention to the design of the computational mesh.

The axi-symmetric flow results predict axi-symmetric screech, which onsets in the flow without artificial 


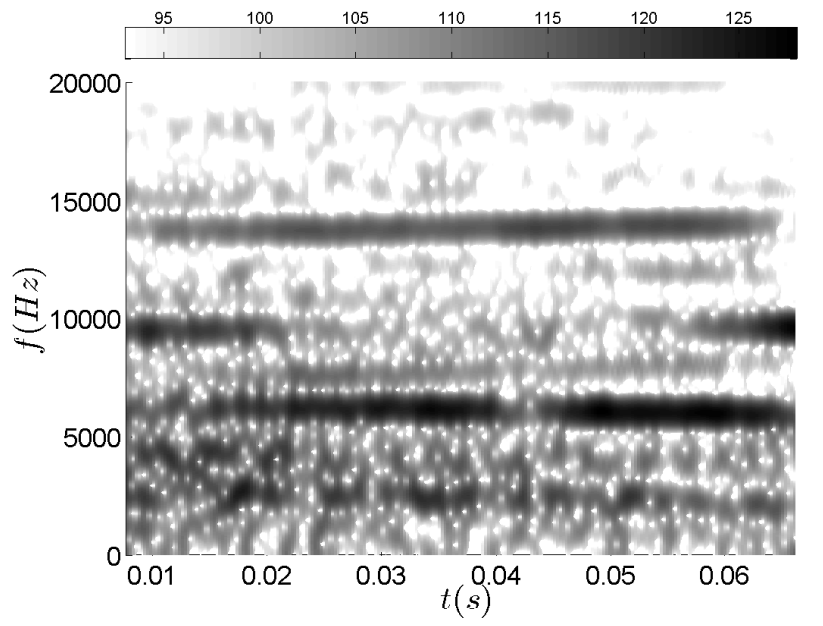

(a) $(x, r)=\left(1.5 D_{e}, 0 D_{e}\right)$.

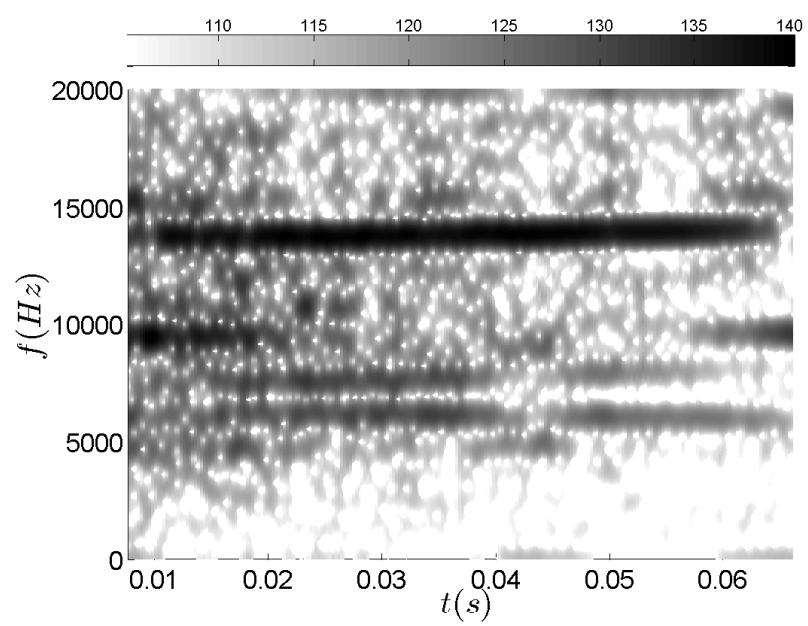

(c) $(x, r)=\left(1.5 D_{e}, 0.5 D_{e}\right)$.

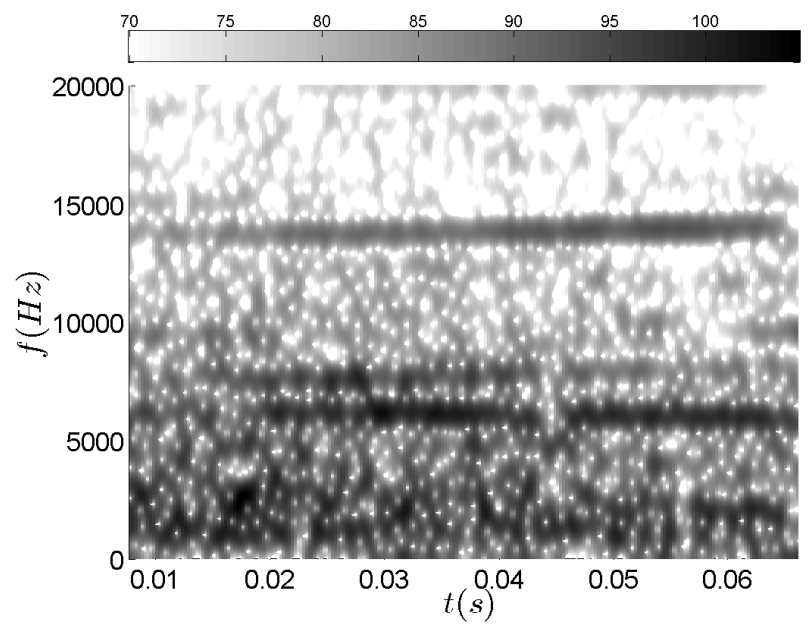

(e) $(x, r)=\left(1.5 D_{e}, 3 D_{e}\right)$.

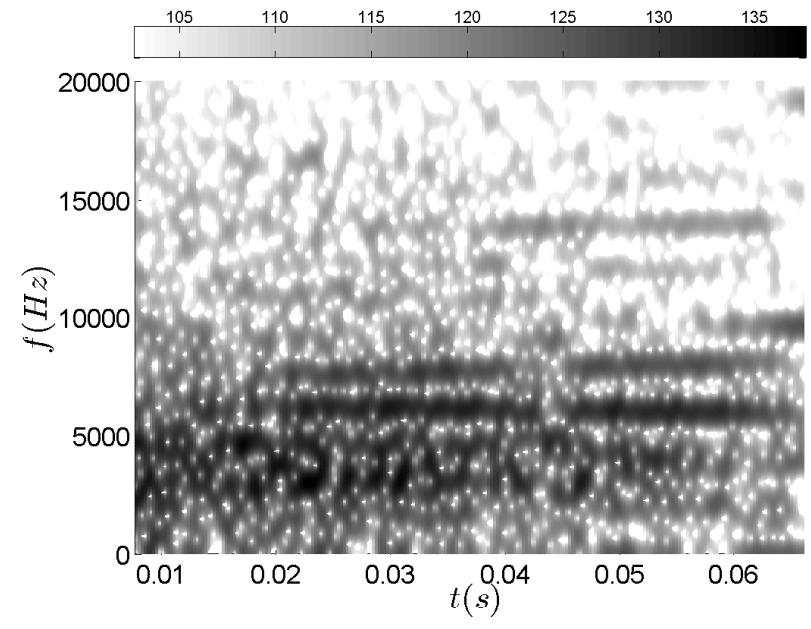

(b) $(x, r)=\left(2.5 D_{e}, 0 D_{e}\right)$.

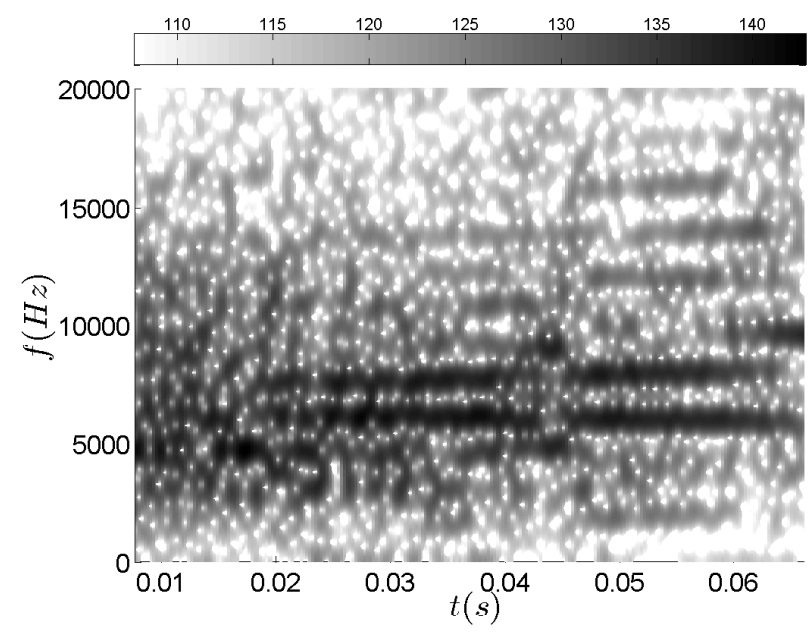

(d) $(x, r)=\left(2.5 D_{e}, 0.5 D_{e}\right)$.

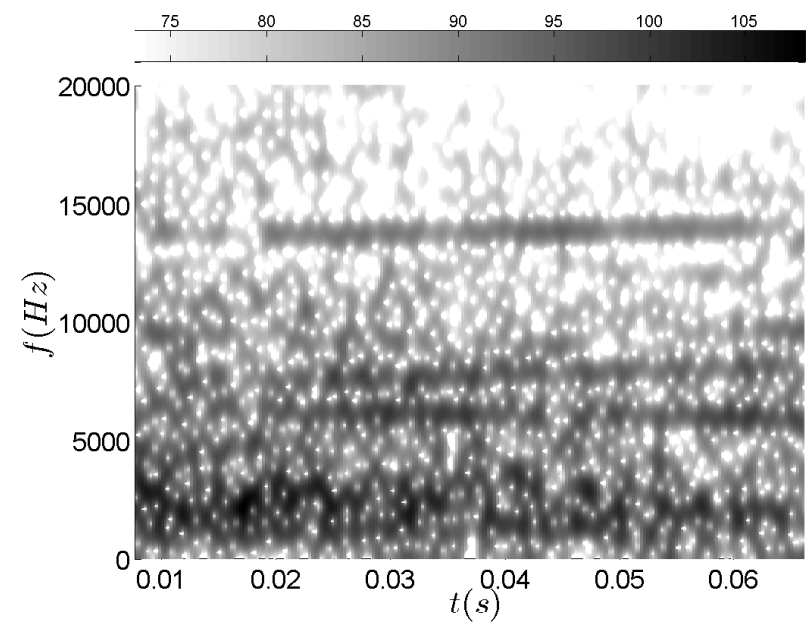

(f) $(x, r)=\left(2.5 D_{e}, 3 D_{e}\right)$.

Figure 7. Iso-levels of predicted aerodynamic pressure (dB re $20 \mu \mathrm{Pa}$ ). 
forcing. This suggests that the model captures the salient time-dependent mechanisms for this self-sustained instability, related to large-scale structures with well-defined narrow-band wavenumber and frequency characteristics.

Under these conditions, time-dependent predictions of the unsteady aerodynamic pressure can be extracted at locations representative of the fuselage position in civil transport aircraft, owing to the use of large-diameter turbofan engines that results in the fuselage distance from the jet being relatively small, when scaled with the bypass nozzle outer diameter.

This confirms that the direct approach for evaluating the forcing pressure field on the fuselage skin, to determine the low-frequency contributions to cabin noise at cruise conditions, is appropriate for this class of aircraft.

\section{Acknowledgements}

The dissemination of these results has received funding from the European Union Seventh Framework Programme FP7/2007-2013 under grant agreement no. 317142. This paper uses digitised experimental data kindly provided by Carlos Pérez-Arroyo and Guillaume Puigt, CERFACS, France.

\section{References}

${ }^{1}$ André, B., 'Etude expérimentale de l'effet du vol sur le bruit de choc de jets supersoniques sous-détendus, Ph.D. thesis, École Centrale de Lyon, Lyon, France, November 2012.

${ }^{2}$ Tam, C. and Hu, F., "On the three families of instability waves of high-speed jets," Journal of Fluid Mechanics, Vol. 201, 1989, pp. 447-483.

${ }^{3}$ Roe, P., "Approximate Riemann solvers, parameter vectors and difference schemes," Journal of Computational Physics, Vol. 43, No. 2, October 1981, pp. 357-372.

${ }^{4}$ Roe, P., "Characteristic-based schemes for the Euler equations," Annual Review of Fluid Mechanics, Vol. 18, 1986, pp. 337-365.

${ }^{5} \mathrm{Hu}, \mathrm{F}$., Hussaini, M., and Manthey, J., "Application of low dissipation and dispersion Runge-Kutta schemes to benchmark problems in computational aeroacoustics," ICASE/LaRC workshop on benchmark problems in computational aeroacoustics $(C A A)$, edited by J. Hardin, J. Ristorcelli, and C. Tam, NASA Conference Publication 3300, National Aeronautics and Space Administration, Hampton, Virginia, May 1995, pp. 73-98.

${ }^{6}$ Rona, A. and Zhang, X., "Time accurate numerical study of turbulent supersonic jets," Journal of Sound and Vibration, Vol. 270, 2004, pp. 297-321.

${ }^{7}$ Pérez-Arroyo, C. and Puigt, G., "Presentation by Early Stage Researcher 3," 2nd Aero TraNet 2 network-wide meeting and seminars by ESRs, INSEAN, Rome, Italy, April 2014.

${ }^{8}$ Raman, G., "Advances in understanding jet screech," Paper 98-0279, AIAA, 36th AIAA Aerospace Sciences Meeting \& Exhibit, Reno, NV, USA, January 1998.

${ }^{9}$ Raman, G., "Advances in understanding supersonic jet screech: Review and perspective," Progress in Aerospace Sciences, Vol. 34, No. 1-2, June 1998, pp. 45-106.

${ }^{10}$ Gharib, M. and Roshko, A., "The effect of flow oscillations on cavity drag," Journal of Fluid Mechanics, Vol. 177, 1987, pp. $44-50$.

${ }^{11}$ Manning, T. and Lele, S., "A Numerical Investigation of Sound Generation in Supersonic Jet Screech," Paper 2000-2081, AIAA, 6th AIAA/CEAS Aeroacoustics Conference, 2000.

${ }^{12}$ Lin, S., Chen, Y., and Shih, S., "Numerical study of MUSCL schemes for computational aeroacoustics," Paper 97-0023, AIAA, 35th Aerospace Sciences Meeting \& Exhibit, Reno, NV, USA, Jan. 1997.

${ }^{13}$ Taylor, J., Jane's all the world's aircraft 1983-84, Jane's Publishing Company Ltd., 238 City Road, London, England, 1983. 